\title{
Successful evolution of a patient with obesity, metabolic syndrome and severe gastro esophageal reflux after probiotic intake
}

\begin{abstract}
Perturbation of the microbiota composition also known as dysbiosis, may play an important role in human health. It is has been observed in obesity, metabolic syndrome, gastrointestinal disturbances among many other conditions. The gram-negative cell wall component Lipopolysaccharide (LPS) translocation might induce metabolic endotoxemia, chronic low-grade inflammation and insulin-resistance.

We report the case of a 52-year-old Caucasian male who suffered severe gastroesophageal reflux, obesity and metabolic syndrome. His condition had worsened several months prior to his first visit as a result of asthenia, morning nausea and abdominal bloating. A high LPS plasma level corroborated the initial suspicion of dysbiosis and endotoxemia.

After 3months of treatment with VSL\#3, a freeze-dried pharmaceutical probiotic containing $112.5 \times 10^{9} \mathrm{CFU} /$ capsule of 3 strains of bifidobacteria, 4 strains of Lactobacillus and Streptococcus salivarius subsp. thermophilus, a remarkable improvement in digestive symptoms, asthenia, cardiometabolic parameters, and anthropometric measures was observed, including weight, fat percentage, waist circumference, blood pressure, lipid profile, glycaemic control, insulin-resistance and plasma LPS level

Due to the significant clinical changes that followed probiotic supplementation, we hypothesize that intestinal dysbiosis plays a crucial role in gastrointestinal symptoms as well as in the severe expression of the comorbidities of our obese patient, probably linked to the proinflammatory state associated to the LPS translocation.
\end{abstract}

Keywords: obesity, metabolic syndrome, dysbiosis, probiotics, microbiota
Volume 2 Issue I - 2015

\author{
Clotilde Vazquez,' Alba Galdon,' Ana I de \\ Cos, ${ }^{2}$ Juan S Chacin,' Isabel Zamarron, ${ }^{3}$ \\ Delia Barrios, ${ }^{3}$ Mercedes Ramirez,' Alfonso \\ Calanas, ${ }^{4}$ Francisco Arrieta $^{3}$ \\ 'Department of Endocrinology and Nutrition, Fundacion \\ Jimenez Diaz_IDCsalud, Spain \\ 2Department of Endocrinology and Nutrition, Hospital La Paz, \\ Spain \\ ${ }^{3}$ Department of Endocrinology and Nutrition, Hospital \\ Universitario Ramon y Cajal, Spain \\ ${ }^{4}$ Department of Endocrinology and Nutrition, Hospital Reina \\ Sofia, Spain
}

Correspondence: Clotilde Vazquez, Department Endocrinology and Nutrition, Fundacion Jimenez Diaz IDCSalud and CIBEROBn, Madrid, Spain, Tel 3491402459I, Email Clotilde.vazquez@fjd.es

Received: December 08, 2014 | Published: January 03, 2015
Abbreviations: LPS, lipopolysaccharide; EDI, eating disorder inventory; GHQ, general health questionnaire; BMI, body mass index

\section{Introduction}

The complex communities of microorganisms that colonize the human gastrointestinal tract play an important role in human health. ${ }^{1-3}$ Perturbation of the microbiota composition, also known as dysbiosis, has been observed in obesity, partly due to a strong decrease in the bacterial diversity. ${ }^{4}$ In addition, the gram-negative cell wall component Lipopolysaccharide (LPS) translocation might induce metabolic endotoxemia, chronic low-grade inflammation, and ultimately insulin resistance. ${ }^{5,6}$ This fact is also related to metabolic syndrome, and eventually to gastroesophageal reflux and esophagitis. Microbes are among the environmental factors that may contribute to the etiology of gastroesophageal reflux and, although little research has been done on the esophageal microbiome, recent data suggest that a scarce presence of the genus Streptococcus and a greater proportion of Gram-negative anaerobes/microaerophiles is primarily correlated with esophagitis.

Probiotics are live microorganisms which once ingested in adequate amounts have beneficial effects on the host. In recent years, probiotics have been shown to have a beneficial effect on obesity and insulin resistance. ${ }^{6,8}$

Here we report a clinical case of an obese patient with severe metabolic syndrome, serious gastroesophageal reflux symptoms, and gut dysbiosis who, following treatment with probiotics, experienced a spectacular improvement in symptoms, endoscopic findings as well as anthropometric measurements and cardiometabolic parameters.

\section{Case report}

A 52-year-old Caucasian male was referred to the outpatient Clinical Nutrition and Obesity consults of the Hospital Ramon y Cajal for obesity treatment prior to anti-reflux surgery. 2years before he had been diagnosed of gastroesophageal reflux disease with hiatal hernia, severe esophagitis and Barrett's esophagus, recurrent dyspeptic symptoms and little response to medical treatment.

As comorbidities of his grade 1 obesity, he also suffered from hypertension, impaired glucose tolerance, non-alcoholic fatty liver disease and dyslipidemia. He was on thyroid hormone replacement therapy for primary hypothyroidism due to Graves' disease posttreatment with radioiodine therapy. He also had diffuse idiopathic skeletal hyperostosis (Forestier's disease), hyper uricemia and gout, which was being treated by the Department of Rheumatology. He didn't take any antidiabetic treatment nor anti-inflammatory drugs.

He referred a progressive weight gain over the previous 2years, which had been attributed to his co-morbidities and their medical treatment. He denied symptoms suggestive of eating disorders, and scored 0 points in the Eating Disorder Inventory (EDI) questionnaire. However his perceived quality of life was poor, scoring 18 points in the Goldberg General Health Questionnaire (GHQ-28).

In the prior 4months, he reported considerable asthenia, a worsening of dyspepsia symptoms, abdominal bloating, early 
morning nausea, occasional vomiting, chronic constipation as well as an increasing self-perception of illness.

At the moment of the first visit, he weighed 97.6kilograms and had a body mass index (BMI) of $33.4 \mathrm{Kg} / \mathrm{m}^{2}$. Even though he was not in acute distress, he presented a congestive appearance with a ketosic-like halitosis and strong corporal odor that reminded the food-fermentation process. Close inspection of his skin revealed mild acanthosis nigricans around his neck and several warty lesions in both temples and cheeks. . Cardiopulmonary auscultation was normal. His abdomen was bloated but soft and non-tender with no visible stretch marks, with three finger-width hepatomegaly. He had mild non-pitting edema in ankles with hardened skin.

His baseline laboratory analysis and endoscopic procedures are shown in Table 1 with the clinical presumption of intestinal dysbiosis, stool and blood samples were obtained. We then prescribed once-daily supplementation with VSL\#3, a freeze-dried pharmaceutical probiotic containing $112.5 \times 10^{9} \mathrm{CFU} /$ capsule of three strains of bifidobacteria: Bifidobacterium longum DSM 24736, Bifidobacterium breve DSM 24732, Bifidobacterium infantis DSM 24737, Lactobacillus acidophilus DSM 24735, Lactobacillus plantarum DSM 24730, Lactobacillus paracasei DSM 24733, Lactobacillus delbrueckii subsp. bulgaricus DSM 24734 and one strain of Streptococcus salivarius subsp. thermophilus DSM 24731

Table I Clinical data of our patient obtained before and after daily probiotic intake

\begin{tabular}{llll}
\hline & Basal & $3^{\text {rd }}$ month & $6^{\text {th }}$ month \\
\hline Anthropometric data & & & \\
\hline Weight $(\mathrm{kg})$ & 97.6 & 92,3 & 89,9 \\
BMI & 33.4 & 31.5 & 30.7 \\
Waist circumference (cm) & 117 & 114 & 113 \\
Fat mass ( kg) & 35.1 & 31.3 & 28.1 \\
Blood pressure & $150 / 99$ & $143 / 103$ & $130 / 8 \mid$ \\
\hline Biochemical data & & & \\
\hline Fast Glycaemia (mg/dl) & 149 & 102 & 116 \\
Plasma insulin uU/ml & 32 & 21 & 16 \\
HOMA-IR & 11.77 & 5.29 & 4.58 \\
Glycated Haemoglobin Alc \% & 6.8 & 6 & 5.6 \\
Total Cholesterol (mg/dl) & 264 & 222 & 215 \\
LDL Cholesterol ( mg/dl) & 179 & 150 & 134 \\
Triglycerides (mg/dl) & 192 & 131 & 134 \\
GPT (U/l) & $7 \mid$ & 36 & 18 \\
LPS (ng/ml) & 0.52 & 0.23 & \\
\hline
\end{tabular}

Three months later, new stool and blood samples were taken. The probiotic treatment was changed to a weakly-basis dose, lasting 3 additional months, after which a new blood test was completed. Conventional fecal cultures were realized by the Department of Microbiology.

The LPS concentrations were determined in $200 \mu 1$ of plasma using a kit based on a Limulus amoebocyte extract (LAL kit endpointQCL1000, Cambrex Bioscience, Walkersville, MD). Careful technique has been performed to avoid environmental or endotoxin contamination. All materials coming in contact with plasma were endotoxin-free.

\section{Results}

Two weeks after initiating the probiotic supplementation, the patient referred a remarkable improvement in both his digestive symptoms and in his quality of life. In the following three months the patient reported a clinically significant improvement in asthenia, the disappearance of both, the morning nausea and vomiting, as well as a normal intestinal daily habit. As a result of this, the patient had stopped all antacids and antireflux medications. His halitosis and the corporal odor had decreased considerably and the skin lesions on his forehead had shrunk. His general health perception had improved, with a GHQ-28 score of 5 .

The patient lost $4 \mathrm{Kg}$, mainly fat, and his waist circumference decreased $3 \mathrm{~cm}$, under balanced diet counseling but without any specific hypocaloric diet An improvement in cardiometabolic parameters was also observed, including blood pressure, lipid profile glycaemic control and insulin or resistance (Table 1). A previous panendoscopy had shown moderate-severe esophagitis (Grade B Los Angeles) with Barrett's image in distal oesophagus, 6months later the procedure revealed an improvement to Mild esophagitis (Grade A Los Angeles), with persistence of Barrett's images.

A considerable decrease in LPS concentration circulating in blood was detected (from 0.52 to $0.23 \mathrm{ng} / \mathrm{ml}$ ) indicating a reduction in bacterial translocation. Microbiological analyses of the stool cultures (before and after probiotic intake) detected normal concentrations of Escherichia coli in the plates and absence of enteropathogens.

\section{Discussion and conclusions}

Even though a great deal of knowledge about probiotics and human health is currently available, there is still much uncertainty regarding the role that specific probiotics can play in certain diseases. ${ }^{9}$

We prescribed this patient standard dietary counseling coupled with a VSL\#3 intake of a multi-species probiotic belonging to lactic acid bacteria. We chose this commercially available preparation for their high content in several Bifidobacterium species and Streptococus termophilus. It has been suggested that in both metabolic syndrome and esophagitis a lower content of these strains is found.

As the case of our patient shows, the prescription of probiotics could be an effective strategy to restore dysbiosis. The severely disturbed digestive functionality of the patient was associated with a particular smell that strongly reminded us of a fermentation tank. He suffered from intense gastroesophageal reflux, esophagitis and Barrett's esophagus. Recent studies of a small number of hosts have shown that nearly 100 Commensal bacterial species reside in the normal distal esophagus. A type I microbiome, dominated by the genus Streptococcus, defines the phenotypically normal esophagus. In contrast, the type II microbiome contains a greater proportion of Gramnegative anaerobes/microaerophiles and is primarily correlated with esophagitis (Odds Ratio: 15.4) and Barrett's esophagus. In addition, it has been found that subjects with low HDL, insulin resistance, and high hsCRP present significantly lower total lactobacilli and bifidobacteria and higher E. coli and bacteroides count. ${ }^{10}$

Our patient's initial levels of LPS were very high, near 100 times the normal value. ${ }^{11,12}$ The treatment was followed by a decrease in half of the circulating LPS concentration, suggesting a better function of the intestinal barrier.

After three months, the patient lost $5.3 \mathrm{Kg}$, mainly from fat and $3 \mathrm{~cm}$ of waist circumference despite his appetite was recovered, his nausea and morning vomiting stopped and he did not follow any specific hypocaloric diet, but only healthy eating recommendations. At the end of 6months-treatment the overall weight loss was close to $8 \mathrm{Kg}$, being 7 from fat mass Although several factors could explain this weight loss, such as the increase in body energy expenditure 
linked to his health-state recovery and a likely improvement of food quality in his daily intake, the contribution of dysbiotic gut microbiota promoting changes in energy extraction from the diet cannot be discarded. We also corroborated an important clinical improvement in his metabolic syndrome parameters: decrease in waist circumference and blood pressure, fat loss with constant lean mass, and increase in metabolic control and quality of life as measured by GHQ-28. It's also worth mentioning the disappearance of the acanthosis nigricans and cutaneous lesions. Taken together, these findings indicate that endotoxin was a trigger factor for metabolic inflammation and insulin resistance. ${ }^{13}$ It has been suggested that changes in the gut microbiota control this process by a mechanism that affects gut barrier function and increases intestinal permeability, which may involve the disruption of the tight junction. Reducing this endotoxin leakage from the gut into the bloodstream, perhaps by modulation of the gut microbiota, could be a target in a strategy to reduce obesity and metabolic disease.

Firmicutes predominance, to the detriment of Bacteroidetes, has been identified in obese patients. ${ }^{14}$ Yadav et al. ${ }^{15}$ recently showed that VSL\#3 in rats may modulate the composition of gut flora (i.e. decreased firmicutes and increased bacteriodetes and bifidobacteria) and stimulate differential production of SCFAs, like butyrate, which have a beneficial effect on weight loss if administered to dietinduced obese mice. It is therefore quite conceivable that the effects of VSL\#3 in our patient could be dependent on the restoration of normal gut microbiota as well as a consequent production of SCFAs. These findings merit further investigation by metagenomic and metabolomics approaches.

In addition, both the hepatomegaly and high GGT levels that we initially identified in this patient were restored after 6 months. A few trials have demonstrated the beneficial effect of some probiotics in nonalcoholic fatty liver disease. ${ }^{16}$

All of these significant clinical changes that followed probiotic supplementation allow us to hypothesize that intestinal dysbiosis plays a crucial role in the severe expression of obese patient's comorbidities, probably linked to the severe proinflammatory state associated to the LPS translocation. A recent randomized trial has shown that VSL\#3 improves metabolic parameters. ${ }^{10}$

The patient's BMI and cardiovascular risk parameters and quality of life are currently excellent; he has discontinued anti-reflux and hypotensive therapy and only maintains thyroxin replacement and VSL\#3 on a weekly basis.

In summary, this is a case of intestinal dysbiosis and endotoxemia treated exclusively with probiotics that objectively shows weight loss, an improvement of severe digestive symptoms and the clinical and biochemical expression of metabolic syndrome. Further research focusing on microbiome and/or metagenomic analysis is needed in order to study the gut microbiota and its modifications after probiotic treatment.

\section{Statement of authorship}

$\mathrm{CV}, \mathrm{AG}$ and JSCh drafted the manuscript. CV performed the discussion, interpretation of the data, and the conclusions, that were enriched by the other authors. All of them gave the final approval of the manuscript.

\section{Acknowledgements}

We acknowledge Rosa Del Campo, Department of Microbiology, Hospital Universitario Ramon y Cajal for her contribution.

\section{Conflict of interest}

The author declares no conflict of interest.

\section{References}

1. Gerritsen J, Hauke Smidt, Ger Rijkers T, et al. Intestinal microbiota in human health. Genes Nutr. 2011;6(3):209-240.

2. Jumpstart Consortium Human Microbiome Project Data Generation Working Group. Evaluation of $16 \mathrm{~S}$ rDNA-Based Community Profiling for Human Microbiome Research. PLoS One. 2012;7(6):e39315.

3. Putignani L, Del Chierico F, Petrucca A, et al. The human gut microbiota: a dynamic interplay with the host from birth to senescence settled during childhood. Pediatr Res. 2014;76(1):2-10.

4. Delzenne NM, Cani PD. Interaction between obesity and the gut microbiota: relevance in nutrition. Annu Rev Nutr. 2011;31:15-31.

5. Jialal I, Rajamani U. Endotoxemia of metabolic syndrome: a pivotal mediator of meta-inflammation. Metab Syndr Relat Disord. 2014;12(9):454-456.

6. Kallio KA, Hatonen KA, Lehto M, et al. Endotoxemia, nutrition, and cardiometabolic disorders. Acta Diabetol. 2015;52(2):395-404.

7. Yang L, Lu X, Nossa CW, et al. Inflammation and intestinal metaplasia of the distal esophagus are associated with alterations in the microbiome. Gastroenterology. 2009;137(2):588-597.

8. Petschow B, Dore J, Hibberd P, et al. Probiotics, prebiotics, and the host microbiome: the science of translation. Ann N Y Acad Sci. 2013;1306:117.

9. Thomas LV, Ockhuizen T, Suzuki K. Exploring the influence of the gut microbiota and probiotics on health: a symposium report. Br J Nutr. 2014;112(Suppl1):1-18.

10. Rajkumar H, Mahmood N, Kumar M, et al. Effect of probiotic (VSL\#3) and omega-3 on lipid profile, insulin sensitivity, inflammatory markers, and gut colonization in overweight adults: a randomized, controlled trial. Mediators Inflamm. 2014;2014:348959.

11. Opal SM, Scannon PJ, Louis Vincent J, et al. Relationship between Plasma Levels of Lipopolysaccharide (LPS) and LPS-Binding Protein in Patients with Severe Sepsis and Septic Shock. J Infect Dis. 1999;180(5):1584-1589.

12. Sun L, Yu Z, Ye X, et al. A Marker of Endotoxemia is associated with Obesity and Related Metabolic Disorders in Apparently Healthy Chinese. Diabetes Care. 2010;33(9):1925-1932.

13. Lee SJ, Bose S, Seo JG, et al. The effects of co-administration of probiotics with herbal medicine on obesity, metabolic endotoxemia and dysbiosis: A randomized double-blind controlled clinical trial. Clin Nutr. 2014;33(6):973-981.

14. Kallus SJ, Brandt LJ. The intestinal microbiota and obesity. J Clin Gastroenterol. 2012;46(1):16-24.

15. Yadav H, Lee JH, Lloyd J, et al. Beneficial metabolic effects of a probiotic via butyrate-induced GLP-1 hormone secretion. J Biol Chem. 2013;288(35):25088-25097.

16. Alisi A, Bedogni G, Baviera G, et al. Randomised clinical trial: the beneficial effects of VSL\#3 in obese children with non-alcoholic steatohepatitis. Aliment Pharmacol Ther. 2014;39(11):1276-1285. 